\title{
Severe immune thrombocytopenic purpura in critical COVID-19
}

\author{
Valérie Lévesque $^{1} \cdot$ Émilie Millaire $^{1} \cdot$ Daniel Corsilli $^{2}$ - Benjamin Rioux-Massé ${ }^{3} \cdot$ François-Martin Carrier ${ }^{2,3,4,5}$ (i)
}

Received: 1 June 2020 / Revised: 18 June 2020 / Accepted: 24 June 2020 / Published online: 1 July 2020

(c) Japanese Society of Hematology 2020

\begin{abstract}
COVID-19 is a new disease with many undescribed clinical manifestations. We report herein a case of severe immune thrombocytopenic purpura (ITP) in a critical COVID-19 patient. A patient presented a severe episode of immune thrombocytopenia $\left(<10 \times 10^{9} / \mathrm{L}\right) 20$ days after admission for a critical COVID-19. This thrombocytopenia was associated with a life-threatening bleeding. Response to first-line therapies was delayed as it took up to 13 days after initiation of intravenous immunoglobulin and high-dose dexamethasone to observe an increase in platelet count. COVID-19 may be associated with late presenting severe ITP. Such ITP may also be relatively resistant to first-line agents. Hematological manifestations of COVID-19, such as the ones associated with life-threatening bleeding, must be recognized.
\end{abstract}

Keywords COVID-19 Thrombocytopenia $\cdot$ Bleeding $\cdot$ Immunoglobulin $\cdot$ Corticosteroids

\section{Introduction}

Many different clinical manifestations of COVID-19 has been described, the most common being a respiratory disease [1]. Hematologic abnormalities are amongst them and thrombocytopenia seems to occur in up to one third of patients [1].

SARS-CoV-1 associated thrombocytopenias were most of the time mild and recovered during the hospital stay [2]. These thrombocytopenias were likely multifactorial. Proposed etiologies in the Severe Acute Respiratory Syndrome (SARS), caused by SARS-CoV-1, included decreased bone marrow production, diffuse endothelial consumption and autoantibodies produced by the immune viral response [2].

François-Martin Carrier

francois.martin.carrier@umontreal.ca

1 Department of Medicine, Université de Montréal, Montréal, Canada

2 Department of Medicine - Critical Care Division, Centre hospitalier de I'Université de Montréal, Montréal, Canada

3 Department of Medicine - Hematology Division, Centre hospitalier de I'Université de Montréal, Montréal, Canada

4 Department of Anesthesiology, Centre hospitalier de I'Université de Montréal, Montréal, Canada

5 Carrefour de I'Innovation, Centre de recherche du Centre hospitalier de I'Université de Montréal (CRCHUM), 900, rue St-Denis, porte S03-434, Montréal, QC H2X 0A9, Canada
Immune thrombocytopenic purpura (ITP) is caused by the production of platelet autoantibodies. ITP may occur after several viral infections, including human immunodeficiency virus (HIV), hepatitis $\mathrm{C}$ virus (HCV), cytomegalovirus, herpes simplex virus, zika virus and others [3]. ITP episodes can vary from being mild to life-threatening. They often require treatment of the underlying infection with appropriate antiviral therapy, when available, in addition to ITP therapies such as intravenous immunoglobulin and glucocorticoids [4].

COVID-19 associated ITP has been recently reported early in the disease course $[5,6]$. We present herein a case of very severe COVID-19 associated ITP that occurred later in the disease course than previously published cases. We obtained consent from the decision surrogate maker to publish this case.

\section{Case report}

A 53-year-old man with well-controlled hypertension, dyslipidemia, type 2 diabetes and a body mass index of 24 presented with a 3-day history of dyspnea, dry cough and fever. His usual medication included Metformin, Rosuvastatin, Irbesartan, Amlodipine and Hydrochlorothiazide. His initial laboratory tests showed a hemoglobin concentration of $117 \mathrm{~g} / \mathrm{L}$ (normal range 130-170), a white blood cell count of $10.2 \times 10^{9} / \mathrm{L}(4.0-10.0)$, a lymphopenia of $0.1 \times 10^{9} / \mathrm{L}$ 
(1.0-4.0), a platelet count of $244 \times 10^{9} / \mathrm{L}(130-400)$, a creatinine of $222 \mu \mathrm{mol} / \mathrm{L}$ (53-112), normal coagulation times and mildly elevated liver enzymes [AST 59 U/L (13-39), ALT 30 U/L (10-39)]. An endotracheal RT-PCR test was positive for SARS-CoV-2.

He quickly developed severe acute respiratory distress syndrome (ARDS), requiring endotracheal intubation. He was mechanically ventilated with lung protective ventilation and prone positioning and was sedated with propofol, fentanyl and cisatracurium infusions. He received ceftriaxone, azithromycin and a thromboprophylaxis with unfractionated heparin. His disease was complicated by acute kidney injury (creatinine rising up to $689 \mu \mathrm{mol} / \mathrm{L}$ ) that required renal replacement therapy and by a methicillin-sensitive staphylococcus aureus ventilator-associated pneumonia treated with a 2-day course of empirical piperacillin-tazobactam and 6 days of cefazolin. On the 20th day, the patient was spontaneously breathing on pressure support ventilation with an inspired concentration of oxygen of $35 \%$. Since he developed ICU-acquired neuromyopathy, a tracheotomy was performed to facilitate weaning of mechanical ventilation. During the procedure, abnormal bleeding from the tracheotomy site and the left main stem bronchus was noticed. We observed afterwards that the platelet count had dramatically fallen from $311 \times 10^{9} / \mathrm{L}$ the day before to $23 \times 10^{9} / \mathrm{L}$ with stable other blood cell counts (Table 1). The patient did not have any skin purpura. However, a head CT scan performed later on hospitalization day 39 showed a small spontaneous intraventricular hemorrhage deemed related to thrombocytopenia.

This severe thrombocytopenia prompted discontinuation of heparin and a change in antibiotics. The patient had normal coagulation times and fibrinogen level and did not present any laboratory signs of hemolysis or microangiopathy. The blood smear was normal other than thrombocytopenia and did not show any schistocyte. An anti-PF4 assay was weakly positive ( 0.72 optical density) but a serotonin release assay came back negative. The ferritin and triglycerides concentrations were mildly elevated. An abdominal computed tomography (CT) scan did not show any hepatomegaly, splenomegaly or lymphadenopathy. Complement dosage was normal and an ADAMTS-13 assay came back negative. Serologies for HIV, HBV and HCV were negative. A bone marrow biopsy or aspiration was deemed uninformative in this context. Our working diagnosis was thus COVID-19 associated ITP.

We administered intravenous immune globulin (IVIG) at a dose of $1 \mathrm{~g}$ per kilogram of body weight daily on ITP days 1 and 2 and a daily dose of $40 \mathrm{mg}$ of intravenous dexamethasone on days 3-6. We also administered several platelet and red blood cell transfusions as well as intravenous tranexamic acid. Due to bleeding and clotting causing complete atelectasis of his left lung and thus severe hypoxemia, he required several daily endobronchial clot removal procedures. The bleeding finally stopped with the aforementioned high platelet transfusion support, although we did not observe any improvement in the platelet count at ITP day 5. In spite of 4 days of optimal first-line therapy, the patient still presented profound thrombocytopenia requiring continuous platelet transfusion support (Table 1). We then decided to proceed with second-line agents and administered romiplostim daily from ITP day 5-14 and a dose of vincristine on ITP day 9. We also administered pulse doses of 500 milligrams of intravenous methylprednisolone daily from ITP days 10-13 (Table 1). We did not consider splenectomy as an appropriate treatment at this stage of the disease. The platelet count started to increase on ITP day 11 and progressively reached $178 \times 10^{9} / \mathrm{L}, 14$ days after first dose of IVIG (Table 1 ).

\section{Discussion}

We believe that severe late ITP associated with COVID19 was the most likely diagnosis to explain the observed isolated fulminant drop in platelet count that caused significant bleeding in this patient. There was no evidence of thrombotic thrombocytopenic purpura, disseminated intravascular coagulation, heparin-induced thrombocytopenia, hemophagocytic lymphohistiocytosis or any lymphoproliferative disorder. The observed thrombocytopenia was relatively resistant to first-line therapies and additional therapies such as romiplostim, an erythropoietin (TPO) receptor agonist, and low-dose vincristine were administered. Thrombocytopenia finally started to improve after 10 days of treatments. Although the patient presented life-threatening bleeding, he improved and survived this episode.

The patient had received several days of penicillin-based treatment and cephalosporins. However, we do not believe that his thrombocytopenia was an adverse effect of the antibiotic treatments due to the rapidity and the severity of the platelet fall. Rare cases of antibiotic associated ITP have been described, but the thrombocytopenia seemed to recover quickly after the agent had been stopped [7]. Besides, cefazolin was not specifically one of them.

Different hematologic abnormalities have been observed in COVID-19 patients. Most of them have resulted in a hypercoagulable state causing thrombotic complications [8]. Nevertheless, mild thrombocytopenia has been observed in up to one third of patients with COVID-19 [1]. A recent study described characteristics of patients presenting with delayed (14 days after symptom onset) SARS-CoV-2 associated thrombocytopenia [9]. Although observed thrombocytopenias were associated with a longer hospital stay and a higher mortality, they were mild and transient (lasting less than 7 days) and not associated with bleeding [9]. These thrombocytopenias were likely multifactorial and etiologies 


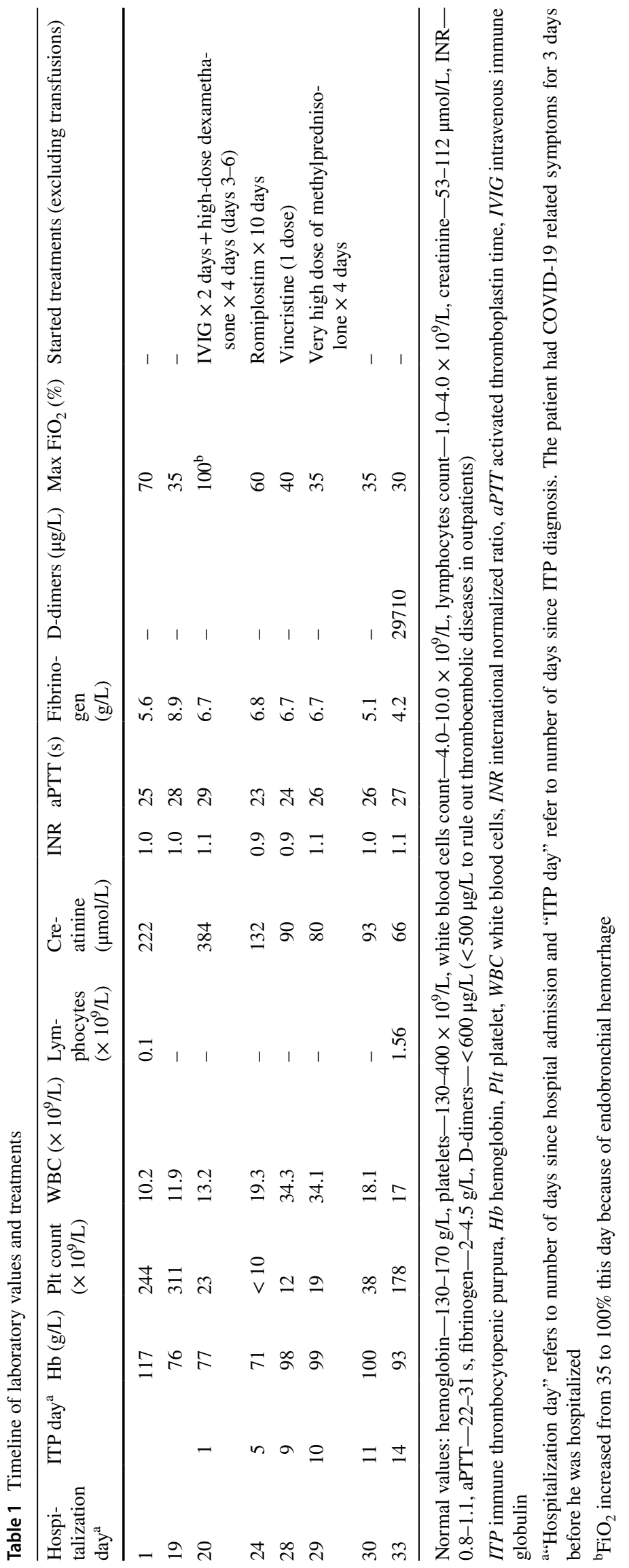


such as decreased bone marrow production and immune destruction were proposed. Most of them were probably not ITP. Such mild thrombocytopenia has also been described in ARDS of pulmonary and extra-pulmonary etiologies and has also been associated with higher mortality [10].

Very few reported cases of hematological manifestations of COVID-19, such as our case, led to severe bleeding complications. Although four cases of COVID-19 ITP have already been reported, most of them occurred early after COVID-19 disease onset and responded well to first-line agents [5, 6]. One of the recently published cases presented a similar thrombocytopenia timeline to our case [6]. This patient presented a severe thrombocytopenia at day 12 of hospitalization while under anticoagulants for a pulmonary embolism for $48 \mathrm{~h}$. He presented an important hemorrhagic episode, only received platelet transfusions to treat thrombocytopenia and died within $24 \mathrm{~h}$, precluding any further observation [6]. Although our case shares with this case the late presentation characteristic, ours is the only one that presented itself with life-threatening bleeding and intracerebral hemorrhage solely due to such a late occurring severe thrombocytopenia. Furthermore, it is the first case of COVID-19 associated ITP showing relative resistance to first-line ITP agents, as all other reported cases presented platelet count improvement within few days after IVIG administration [5, 6]. Although we administered therapies that have been previously used to increase platelet production in refractory cases that are at high risk of bleeding, we believed we observed a late response to first-line agents, since second-line agents did not have time to show any significant clinical effect $[4,11]$.

ITP is not commonly associated with severe bleeding, as only $10 \%$ of adult patients with ITP presented any severe non-intracranial hemorrhage and $2 \%$ an intracerebral hemorrhage in a recent review [12]. Amongst reported COVID-19 associated ITP cases, including ours, bleeding is frequently reported $[5,6]$. This observation may be either related to specific pathophysiological characteristics of the disease or to a reporting bias but needs further attention. We also observed a relative time lag in the clinical response to ITP first-line agents in our case. This relative lag may be caused by the underlying disease severity and its associated high antibody load but may also be related to the ongoing bleeding that may have contributed to platelet consumption and loss of the administered IVIG. Although it is not unusual for ITP to start to improve after 7-10 days of treatment, such a late response is not common [4].

As in many cases of ITP, there is no absolute proof that a specific etiology is the true causal factor for the immune reaction. However, several viral infections have been associated with ITP in the past [3]. In COVID-19, disruption of the immune response is thought to play an important role in the disease, although the pathophysiology is yet to be better understood [13]. A better understanding of such immune pathway will be essential to define best therapeutic approaches to ITP, either associated or not with COVID-19, in the context of the pandemic [13].

\section{Conclusion}

Our case of ITP presented itself late after the COVID-related classical clinical manifestations began, was not associated with any skin manifestations of ITP, presented severe hemorrhages and was relatively resistant to first-line agents. To our knowledge, our case is the first severe ITP occurring late in the disease process of COVID-19, associated with severe bleeding and relatively resistant to IVIG treatment. This case suggests that hematologic complications may occur in COVID-19 and cause life-threatening bleeding complications. Such manifestations must be included in the clinical evaluation of patients infected by SARS-CoV-2.

Author contributions VL, EM, DC, BRM and FMC took care of the patient and participated in the conception of the study, the analysis of the data and writing of the manuscript.

Funding FMC is a recipient of a Career Award from the Fonds de Recherche du Québec - Santé.

\section{Compliance with ethical standards}

Conflict of interest All authors declare they have no competing interests.

\section{References}

1. Guan W-J, Ni Z-Y, Hu Y, Liang W-H, Ou C-Q, He J-X, et al. Clinical characteristics of coronavirus disease 2019 in China. N Engl J Med. 2020;382(18):1708-20.

2. Yang M, Ng MHL, Li CK. Thrombocytopenia in patients with severe acute respiratory syndrome. Hematology. 2005;10(2):101-5.

3. Liebman HA. Viral-associated immune thrombocytopenic purpura. Hematol Am Soc Hematol Educ Program. 2008;1:212-8.

4. Neunert C, Terrell DR, Arnold DM, Buchanan G, Cines DB, Cooper N, et al. American society of hematology 2019 guidelines for immune thrombocytopenia. Blood Adv. 2019;3(23):3829-66.

5. Zulficar AA, Lorenzo-Villalba N, Hassler P, Andrès E. Immune throbocytopenic purpura in a patient with COVID-19. N Engl J Med. 2020;383(18):e43.

6. Bomhof G, Mutsaers PGNJ, Leebeek FWG, Boekhorstte PAW, Hofland J, Croles FN, et al. COVID-19-associated immune thrombocytopenia. Br J Haematol. 2020. (published online 2020 May 18).

7. Arnold DM, Kukaswadia S, Nazi I, Esmail A, Dewar L, Smith JW, et al. A systematic evaluation of laboratory testing for drug-induced immune thrombocytopenia. J Thromb Haemost. 2013;11(1):169-76.

8. Helms J, Tacquard C, Severac F, Leonard-Lorant I, Ohana M, Delabranche X, et al. High risk of thrombosis in patients in severe 
SARS-CoV-2 infection: a multicentre prospective cohort study. Intensive Care Med. 2020;46(6):1089-1098.

9. Chen W, Li Z, Yang B, Wang P, Zhou Q, Zhang Z, et al. Delayedphase thrombocytopenia in patients of coronavirus disease 2019 (COVID-19). Br J Haematol. 2020. (published online 2020 May 26).

10. Wang T, Liu Z, Wang Z, Duan M, Li G, Want S, et al. Thrombocytopenia is associated with acute respiratory distress syndrome mortality: an international study. PLoS One. 2014;9(4):e94124.

11. Samson M, Fraser W, Lebowitz D. Treatments for primary immune thrombocytopenia: a review. Cureus. 2019;11(10):e5849.

12. Neunert C, Noroozi N, Norman G, Buchanan GR, Goy J, Nazi I, et al. Severe bleeding events in adults and children with primary immune thrombocytopenia: a systematic review. J Thromb Haemost. 2015;13(3):457-64.

13. Pavord S, Thachil J, Hunt B, Murphy M, Lowe G, Laffan M, et al. Practical guidance for the management of adults with Immune Thrombocytopenia during the COVID-19 pandemic. Br J Haematol. 2020;189(6):1038-1043.

Publisher's Note Springer Nature remains neutral with regard to jurisdictional claims in published maps and institutional affiliations. 